\title{
Noninteracting Force/Motion Control of Defective Manipulation Systems
}

\author{
D. Prattichizzo ${ }^{\dagger} \quad$ P. Mercorelli ${ }^{\ddagger} \quad$ A. Bicchi ${ }^{\dagger} \quad$ A. Vicino ${ }^{\ddagger}$ \\ †Dipartimento di Sistemi Elettrici e Automazione, Università di Pisa \\ via Diotisalvi, 2, 56100 Pisa, Italia. domenico@dsea.unipi.it, bicchi@dsea.unipi.it \\ $\ddagger$ Facoltà di Ingegneria, Università di Siena \\ via Roma, 56, 53100 Siena, Italia. mercorelli@unisi.it, vicino@unisi.it
}

\begin{abstract}
This paper deals with the problem of noninteracting force/motion control for manipulation systems with possible kinematic defectivity. A geometric approach is adopted in the paper. The main result of the paper shows that a suitable choice of the outputs exists, for which a structural noninteraction property holds, and such that most practical manipulation tasks can be naturally specified.
\end{abstract}

\section{Introduction}

The coordinated use of multiple fingers in a robot hand or, similarly, of multiple arms in cooperating tasks; the use of the inner links of a robot arm or finger to hold an object, and the exploitation of parallel mechanical structures, are examples of non-conventional usage of mechanisms for manipulation. We will refer to such devices as "general manipulation systems".

The aspect of general manipulation systems that our analysis is most focused on is defectivity of their kinematics. In many devices whose design is inspired to the pursuit of the least complex solution to a given class of manipulation tasks, a "defect" of the number of control variables with respect to the problem dimensionality frequently arises.

The main goal of dexterous manipulation tasks consists of controlling the motion of the manipulated object along with the grasping forces exerted on the object. In the robotics literature, the general problem of force/motion control is known as "hybrid control". For a broad overview of the manipulation control problem, the reader is referred to [12] and the references therein.

The present paper is aimed at the synthesis of a noninteracting control law with respect to the rigid-body object motions and the reachable contact forces along with the possible mechanism redundancy. The struc0-7803-3590-2/96 \$5.00 $\odot 1996$ IEEE tural decoupling of motion and force control in manipulation is of paramount importance whenever advanced robotic applications are considered, e.g. surgical applications or high precision tasks of micro-manipulation.

In this paper a geometric approach (cf. [1], [2], [14], [7]) to the control problem is adopted.

We make use of a linearized model at an equilibrium configuration of the general manipulation mechanisms and we prove that the local noninteraction of "rigidbody" object motions and reachable "internal" forces is a structural property of general manipulation systems. The use of linearized model dynamics in the analysis of general manipulation systems is believed to be a significant advancement with respect to the literature, which is almost solely based on quasi-static models, expecially for defective systems, and in fact provides richer results and better insight. Furthermore, the linearized analysis is considered as a fundamental preparatory step towards full nonlinear analysis, which at the moment appears to be too complex to achieve in full generality. Finally, it is worth while to mention that there exists a subclass of cartesian manipulators where the linearized model provides an exact model of the whole system dynamics.

\section{Dynamic model}

The linearized model of the dynamics of a manipulation system is derived. For a detailed discussion of this model refer to [9] and [5].

We denote by $\mathbf{q} \in \mathbb{R}^{q}$ the vector of manipulator joint positions, $\tau \in \mathbb{R}^{q}$ the vector of joint actuator torques, $\mathbf{u} \in \mathbb{R}^{d}$ the vector locally describing the position and the orientation of a frame attached to the object, and $\mathbf{w} \in \mathbb{R}^{d}$ the vector of forces and torques resultant from external forces acting directly on the object. In the literature, $\mathbf{w}$ is usually referred to as the disturbance vector. 


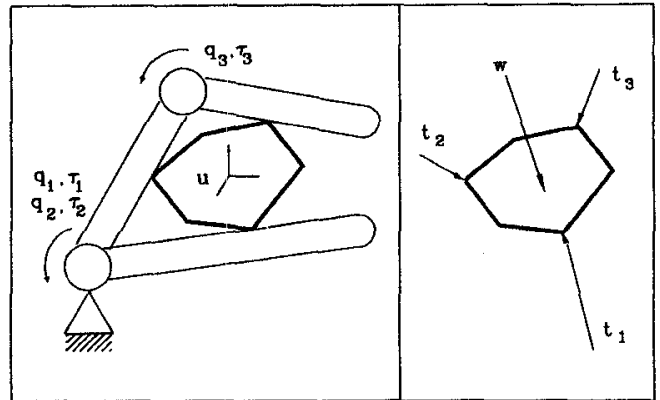

Figure 1: Vector notation for general manipulation system analysis.

The force/torque interaction $\mathbf{t}_{i}$ (fig. 1) at the $i$-th contact is taken into account by using a lumped-parameter $\left(\mathbf{K}_{i}, \mathbf{B}_{i}\right)$ model of visco-elastic phenomena. According to this model, the contact force vector $t_{i}$ is

$$
\mathbf{t}_{i}=\mathbf{K}_{i}\left({ }^{h} \mathbf{c}_{i}-{ }^{o} \mathbf{c}_{i}\right)+\mathbf{B}_{i}\left({ }^{h} \dot{\mathbf{c}}_{i}-{ }^{o} \dot{\mathbf{c}}_{i}\right)
$$

where vectors ${ }^{h} \mathbf{c}_{i}$ and ${ }^{\circ} \mathbf{c}_{i}$ describe the postures of two contact frames, the first on the manipulator and the second on the object, where the $i$-th contact spring and damper are anchored. Matrices $\mathbf{K}_{i}$ and $\mathbf{B}_{i}$ are symmetric and positive definite (p.d.) and their dimension depend on the particular model used to describe the contact interaction (cf. [11]). To compact notation, contact forces and contact points are grouped into vectors $\mathbf{t},{ }^{h} \mathbf{c}$ and ${ }^{\circ} \mathbf{c}$. Similarly, $\mathbf{K}_{i}$ 's and $\mathbf{B}_{i}$ 's are grouped to build the grasp stiffness and damping symmetric and p.d. matrices $\mathbf{K}$ and $\mathbf{B}$.

The Jacobian $\mathbf{J}$ and grasp matrix $\mathbf{G}$ are defined by the linear maps relating the velocities of vectors ${ }^{h} \mathbf{c}$ and ${ }^{o} \mathbf{c}$ with the joint and object velocities $\dot{q}$ and $\dot{\mathbf{u}}$, respectively:

$$
{ }^{h} \dot{\mathbf{c}}=\mathbf{J} \dot{\mathbf{q}} ; \quad{ }^{o} \dot{\mathbf{c}}=\mathbf{G}^{T} \dot{\mathbf{u}} .
$$

Note that, dually, $\mathbf{J}^{T} \mathbf{t}$ and $\mathbf{G t}$ represent the effects of contact forces $\mathbf{t}$ on the manipulation and object dynamics whose nonlinear models are:

$$
\begin{aligned}
& \mathbf{M}_{h} \ddot{\mathbf{q}}+\mathbf{Q}_{h}=-\mathbf{J}^{T} \mathbf{t}+\tau ; \\
& \mathbf{M}_{o} \ddot{\mathbf{u}}+\mathbf{Q}_{o}=\mathbf{G} \mathbf{t}+\mathbf{w} .
\end{aligned}
$$

Here, $\mathbf{M}_{h}$ and $\mathbf{M}_{o}$ are inertia symmetric and p.d. matrices, while $\mathbf{Q}_{h}$ and $\mathbf{Q}_{o}$ are terms including velocitydependent and gravity forces of the manipulator and of the object, respectively.

Let $\mathbf{q}=\mathbf{q}_{o}, \mathbf{u}=\mathbf{u}_{o}, \dot{\mathbf{q}}=\dot{\mathbf{u}}=\mathbf{0}, r=\tau_{o}\left(=\mathbf{J}^{T} \mathbf{t}_{o}\right)$, $\mathbf{w}=\mathbf{w}_{o}\left(=-\mathbf{G t}_{o}\right), \mathbf{t}=\mathbf{t}_{o}$, be a reference equilibrium configuration, the linear approximation of the dynamics in the neighbourhood of such equilibrium point is given by

$$
\dot{\mathbf{x}}=\mathbf{A} \mathbf{x}+\mathbf{B}_{\tau} \delta \tau+\mathbf{B}_{w} \delta \mathbf{w}
$$

where state, input and disturbance vectors are defined as the departures from the reference equilibrium point:

$$
\begin{aligned}
\mathbf{x} & =\left[\delta \mathbf{q}^{T}, \delta \mathbf{u}^{T}, \delta \dot{\mathbf{q}}^{T}, \delta \dot{\mathbf{u}}^{T}\right]^{T}= \\
& =\left[\left(\mathbf{q}-\mathbf{q}_{o}\right)^{T}\left(\mathbf{u}-\mathbf{u}_{o}\right)^{T} \dot{\mathbf{q}}^{T} \dot{\mathbf{u}}^{T}\right]^{T} ; \\
\delta \tau & =\tau-\mathbf{J}^{T} \mathbf{t}_{o} ; \\
\delta \mathbf{w} & =\mathbf{w}+\mathbf{G} \mathbf{t}_{o}
\end{aligned}
$$

and the dynamic, input and disturbance matrices $\mathbf{A}$ $\mathbf{B}_{\tau}$ and $\mathbf{B}_{w}$ are

$$
\left[\begin{array}{cc}
\mathbf{0} & \mathbf{1} \\
\mathbf{L}_{k} & \mathbf{L}_{b}
\end{array}\right] ;\left[\begin{array}{c}
\mathbf{0} \\
\mathbf{0} \\
\mathbf{M}_{h}^{-1} \\
\mathbf{0}
\end{array}\right] ;\left[\begin{array}{c}
\mathbf{0} \\
\mathbf{0} \\
\mathbf{0} \\
\mathbf{M}_{0}^{-1}
\end{array}\right],
$$

respectively. To simplify notation we will henceforth omit the symbol $\delta$. Assuming a locally isotropic model of visco-elastic phenomena, and assuming that gravity and local variations of the Jacobian and grasp matrices are negligible, all the dynamic contributions of terms $\mathbf{Q}_{h}$ and $\mathbf{Q}_{o}$ can be neglected and simple expressions are obtained for the blocks $\mathbf{L}_{k}$ and $\mathbf{L}_{b}$, as $\mathbf{L}_{k}=-\mathbf{M}^{-1} \mathbf{P}_{k}$, and $\mathbf{L}_{b}=-\mathbf{M}^{-1} \mathbf{P}_{b}$ where $\mathbf{M}=\operatorname{diag}\left(\mathbf{M}_{h}, \mathbf{M}_{o}\right)$, and

$$
\begin{aligned}
& \mathbf{P}_{k}=\left[\begin{array}{c}
\mathbf{J}^{T} \\
-\mathbf{G}
\end{array}\right] \mathbf{K}\left[\begin{array}{ll}
\mathbf{J} & -\mathbf{G}^{T}
\end{array}\right] ; \\
& \mathbf{P}_{b}=\left[\begin{array}{c}
\mathbf{J}^{T} \\
-\mathbf{G}
\end{array}\right] \mathbf{B}\left[\begin{array}{ll}
\mathbf{J} & -\mathbf{G}^{T}
\end{array}\right] .
\end{aligned}
$$

The following grasp properties, based on matrices $\mathbf{J}$ and $\mathbf{G}$, have a relevant influence on the dynamic behaviour of the manipulation system.

Definition 1 A grasp (or manipulation system) is said "defective" if $\operatorname{ker}\left(\mathbf{J}^{T}\right) \neq \mathbf{0}$.

From (3) $\mathbf{J}^{T} \in \mathbb{R}^{(q \times t)}$ where $t$ is the number of components of the contact force vector $\mathbf{t}$. Thus, whenever the manipulation system has less degrees of freedom (DoF's) $q$ than $t$, it exhibits a defective grasp. When the system is defective, there exists directions for $t$ which do not influence manipulator dynamics (3). Such a scenario may be considered as a common factor of all defective manipulation systems and this is due to their intrinsically low number of DoF's. The reader is referrd to e.g. [8] for a more detailed discussion of defectivity.

Definition $2 A$ grasp is said "indeterminate" if $\operatorname{ker}\left(\mathbf{G}^{T}\right) \neq 0$.

If the grasp is indeterminate, there exist motions of the objects under which no variations of contact force occur (2). In other words, indeterminacy implies that the object is not firmly grasped.

Definition 3 A manipulation system is said "graspable" if $\operatorname{ker}(\mathbf{G}) \neq 0$. 
If the system is graspable it is possible to exert contact forces with zero resultant forces on the object. Usually in the literature the forces belonging to the null space of $\mathbf{G}$ are referred to as "internal forces". Such forces play a fundamental role in controlling the manipulation task. It is intuitive that, without internal forces squeezing the object, a manipulator only accommodates the object, rather than grasping it. Whenever the effect of a disturbance action on the object is in the tangential direction of a manipulator contact, the system cannot reject such a disturb by simply opposing a contact force. It must generate an additional internal force to keep the total contact force in the friction cone and to keep the contact.

Finally, the well-known notion of manipulator redundancy is formalized as follows.

Definition 4 A grasp is said "redundant" if $\operatorname{ker}(\mathbf{J}) \neq$ 0 .

The concept of general manipulation system is formalized as follows: every manipulation system, in a given grasp configuration, defective or not, indeterminate or not, graspable or not and finally redundant or not is referred to as a "general" manipulation system. In other words the generality of a manipulation system is related to its Jacobian and grasp matrices.

As far as stabilizability of the linearized dynamics is concerned we report the following proposition stated in $[8]$

Proposition 1 If the system is not indeterminate. i.e. $\operatorname{ker}\left(\mathbf{G}^{T}\right)=0$. then the minimal $\mathbf{A}$-invariant subspace containing the $\operatorname{im}\left(\mathbf{B}_{\tau}\right)$, $\min \mathcal{I}\left(\mathbf{A}, \mathbf{B}_{\tau}\right)$, is externally stable.

From now on we will assume that there is not indeterminacy, i.e. $\operatorname{ker}\left(\mathbf{G}^{T}\right)=\mathbf{0}$. Such an assumption is needed for the stabilizability of the manipulation system dynamics (4).

\section{Force/motion control}

The main goal of manipulation tasks consists of controlling the motion of the manipulated object. The stimulating aspect of manipulation control is that the manipulated object is not anchored to the robotic device, but this one acts on the object through passive (not directly actuated) "joints" consisting of a mechanical unilateral contact. Since contact constraints ensure both the object grasp and motion control. their nonviolation is of paramount importance.

Assuming that a general task specification is given in terms of object motion. the remaining degrees of freedom by which contact phenomena can be controlled

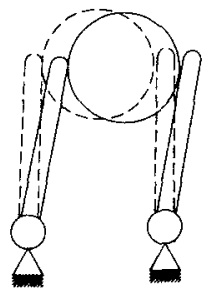

Figure 2: Representative motions for the subspace $\operatorname{im}\left[\Gamma_{q c}^{T} \Gamma_{u s}^{T}\right]^{T}$.

correspond to the "internal forces". These forces belong to the null space of the grasp matrix $\mathbf{G}$ and, as already pointed out, they are called "internal" as their resultant action on the object dynamics is null.

In order to pursue our investigation into force/motion control, the outputs of the dynamic system (4) must be defined. In the spirit of [8] we consider the "rigidbody coordinate object motions", the "reachable internal contact forces" and the "manipulator dynamic redundancy".

\subsection{Rigid-body coordinate object motions}

Rigid-body kinematics are of particular interest in the control of manipulation systems. Rigid-body kinematics have been studied in a quasi-static setting in [4] and in terms of unobservable subspaces in [5]. In both cases rigid kinematics were described by a matrix $\Gamma$ whose columns form a basis for $\operatorname{ker}\left[\mathbf{J}-\mathbf{G}^{T}\right]=\mathrm{im}(\boldsymbol{\Gamma})$ where

$$
\begin{aligned}
& \boldsymbol{\Gamma}=\left[\begin{array}{ccc}
\boldsymbol{\Gamma}_{r} & \boldsymbol{\Gamma}_{q c} & \mathbf{0} \\
\mathbf{0} & \boldsymbol{\Gamma}_{u c} & \boldsymbol{\Gamma}_{i}
\end{array}\right] \\
& \text { with } \mathbf{J} \boldsymbol{\Gamma}_{q c}=\mathbf{G}^{T} \boldsymbol{\Gamma}_{u c}
\end{aligned}
$$

being $\boldsymbol{\Gamma}_{r}$ a basis matrix (b.m.) of the subspace of redundant manipulator motions $\operatorname{ker}(\mathbf{J}) . \boldsymbol{\Gamma}_{i}$ a b.m. of the subspace of indeterminate object motions $\operatorname{ker}\left(\mathbf{G}^{T}\right)$. and $\boldsymbol{\Gamma}_{q r}$ and $\boldsymbol{\Gamma}_{u c}$ conformal partitions of a complementary basis matrix ${ }^{1}$ (c.b.m.).

The column space of $\boldsymbol{\Gamma}_{\mathrm{c}}=\left[\begin{array}{l}\boldsymbol{\Gamma}_{q} \\ \boldsymbol{\Gamma}_{u e}\end{array}\right]$ consists of coordinated rigid-body motions of the mechanism. for the manipulator $\left(\boldsymbol{\Gamma}_{g c}\right)$ and the object $\left(\boldsymbol{\Gamma}_{u c}\right)$ components. Physically rigid-body displacements are those that do not involve variation of contact forces, from which the name 'rigid'. Figure 2 shows such subspaces for a simple devices.

The following proposition. proven in [5]. shows that rigid-body motions are reachable. i.e. they belong to the space of reachability of linear system (4) with input the vector of joint torques $\tau$.

\footnotetext{
${ }^{1} W$ is called a complementary basis matrix of $V$ to $f$ if it is f.c.r. and im $(\mathbf{W})=V=A$.
} 
Proposition 2 Let the subspace of rigid-body positions and velocities be defined as the column space of $\mathbf{T}_{c}$, where

$$
\mathbf{T}_{c}=\left[\begin{array}{cc}
\boldsymbol{\Gamma}_{q c} & \mathbf{0} \\
\boldsymbol{\Gamma}_{u c} & \mathbf{0} \\
\mathbf{0} & \boldsymbol{\Gamma}_{q c} \\
\mathbf{0} & \boldsymbol{\Gamma}_{u c}
\end{array}\right],
$$

then it holds $\operatorname{im}\left(\mathbf{T}_{c}\right) \subseteq \min \mathcal{I}\left(\mathbf{A}, \mathbf{B}_{\tau}\right)$.

Notice that the rigid-body subspace is only a subspace of the reproducible one which also contains motions due to deformations of elastic elements in the model.

Rigid-body kinematics are of particular interest in the control of manipulation systems. Since they do not involve visco-elastic deformations of bodies, they can be regarded as low-energy motions. In a few words, they represent the easiest way to move the object.

The object-position regulated output $\mathbf{e}_{u c}$ is chosen as the projection, through $\boldsymbol{\Gamma}_{u c}^{P}$, of object positions $\mathbf{u}$ onto the subspace of rigid-body object motions $\operatorname{im}\left(\boldsymbol{\Gamma}_{u c}\right)$ :

$$
\begin{aligned}
& \mathbf{e}_{u c}=\mathbf{E}_{u c} \mathbf{x} ; \quad \text { with } \mathbf{E}_{u c}=\Gamma_{u c}^{P}\left[\begin{array}{llll}
\mathbf{0} & \mathbf{I} & 0 & 0
\end{array}\right] \\
& \text { where } \boldsymbol{\Gamma}_{u c}^{P}=\boldsymbol{\Gamma}_{u c}\left(\boldsymbol{\Gamma}_{u c}^{T} \boldsymbol{\Gamma}_{u c}\right)^{-1} \boldsymbol{\Gamma}_{u c}^{T} \text {. }
\end{aligned}
$$

\subsection{Reachable internal contact forces}

The control of contact forces $\mathbf{t}$ is a fundamental part of the manipulation control problem. Contact forces are able to maintain the grasp, to reject disturbance wrenches $\mathbf{w}$ and to control the object motion. In [9] the reachable subspace of contact forces as outputs of the dynamic system. (4) was studied. The main relevant result is reported in the next proposition.

Let us define $\delta \mathbf{t}$ as the departures of contact force vector $\mathbf{t}$ from the reference equilibrium $\mathbf{t}_{o}(5)$. Its first order approximation can be easily evaluated by substituting differential kinematics (2) in $\mathbf{t}$, the grouped vector of $\mathbf{t}_{i}$ 's (1). Hence $\mathbf{t}=\mathbf{C}_{t} \mathbf{x}$ where $\mathbf{C}_{t}=\left[\mathbf{K J}-\mathbf{K} \mathbf{G}^{T} \mathbf{B J}-\mathbf{B G}^{T}\right]$.

We assume that stiffness matrix $\mathbf{K}$ and damping matrix $\mathbf{B}$ are proportional (cf. [6]). Such an assumption allows us to extend easily some results obtained in previous works ([9] and [5]), to the cases where $\mathbf{B} \neq \mathbf{0}$. Under the assumption of proportionality, those geometric results depend only on the $\operatorname{im}(\mathbf{K})=i m(\mathbf{B})$, thus we will henceforth disregard the explicit dependence on $\mathbf{B}$.

\section{Proposition 3}

The reachable subspace of contact forces $\mathbf{t}$ is $\mathcal{R}_{t, \tau}=$ $\mathbf{C}_{t} \min \mathcal{I}\left(\mathbf{A}, \mathbf{B}_{\tau}\right)=\min \mathcal{I}\left(\mathbf{K G}^{T} \mathbf{M}_{o}^{-1} \mathbf{G}, \mathbf{K J}\right)$

In this work we are interested in controlling those contact forces belonging to the null space of the grasp matrix $\mathbf{G}$. In general the null space of $\mathbf{G}$ is not completely reachable. The importance of the reachability of internal forces in grasping was clarified in [3], where the principle of virtual work was used to describe the subspace of active internal forces, and in [8] where the asymptotically reachable internal forces were studied as steady state behaviour of a suitable transfer function. Here we want to characterize the reachable internal forces subspace $\mathcal{R}_{t i, \tau}$ as the intersection:

$$
\mathcal{R}_{t i, \tau}=\mathcal{R}_{t, \tau} \cap \operatorname{ker}(\mathbf{G})
$$

The following theorem, proven in [10], provides an explicit formula for the reachable internal forces subspace:

\section{Theorem 1}

$$
\begin{aligned}
& \mathcal{R}_{t i, r}=\operatorname{im}\left(\mathbf{P}_{N G} \mathbf{C}_{t}\right)=\operatorname{im}\left(\mathbf{P}_{N G} \mathbf{K J}\right) \\
& \text { with } \mathbf{P}_{N G}=\mathbf{I}-\mathbf{K G}^{T}\left(\mathbf{G K G} \mathbf{G}^{T}\right)^{-1} \mathbf{G} .
\end{aligned}
$$

According to this result, the subspace of reachable internal forces is obtained by the projector on the null space of $\mathbf{G}, \mathbf{P}_{N G}$, acting on the column space of $\mathbf{C}_{t}$. Notice that Theorem 1 states the equality of $\mathcal{R}_{t i, \tau}$ with the active forces in [3] and with the asymptotically reachable forces in $[8]$.

In order to specify consistent control outputs, we follow the suggestion of Theorem 1 and choose as regulated force output $\mathbf{e}_{t i}$ the projection of the contact force vector $\mathbf{t}$ on the null space of $\mathbf{G}$, i.e. the reachable internal contact forces:

$$
\begin{aligned}
& \mathbf{e}_{t i}=\mathbf{E}_{t i} \mathbf{x} ; \text { with } \mathbf{E}_{t i}=\mathbf{P}_{N G} \mathbf{C}_{t}=\left[\begin{array}{llll}
\mathbf{Q} & \mathbf{0} & \mathbf{Q} & \mathbf{0}
\end{array}\right] \\
& \text { where } \mathbf{Q}=\left(\mathbf{I}-\mathbf{K G}^{T}\left(\mathbf{G} \mathbf{K G}^{T}\right)^{-1} \mathbf{G}\right) \mathbf{K J} \text {. }
\end{aligned}
$$

\section{Noninteraction as a structural property}

The present section is aimed at the analysis of the noninteracting control property for grasping mechanisms with respect to the rigid-body object motions and the reachable contact forces together with the possible mechanism redundancy. The geometric approach is used in such analysis. It should be remarked that the earliest geometric approaches to noninteracting control are due to Basile and Marro ([1], [2]) and to Wonham and Morse ([14], [7] [13]). The result of this section regards the local force/motion noninteracting control of general manipulation mechanisms and is based on necessary and sufficient conditions for the existence of the noninteraction control law given in [2] and [1].

Before attacking the problem of the structural noninteraction, let us introduce the third output vector $\mathbf{e}_{q r}$ to take into account the possible redundancy of the mechanism. Whenever the analysis is not static, the inertia matrix $\mathbf{M}_{h}$ play a key role in characterizing the redundance displacements of the manipulator. Therefore, we define the redundancy output matrix $\mathbf{E}_{q r}$ as

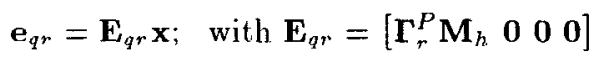

where $\Gamma_{r}^{P}$ is the projection matrix onto $\operatorname{ker}(\mathbf{J})$ whose b.m. is $\boldsymbol{\Gamma}_{r}$

$$
\Gamma_{r}^{P}=\Gamma_{r}\left(\Gamma_{r}^{T} \Gamma_{r}\right)^{-1} \Gamma_{r}^{T}
$$


Definition 5 A control law for the dynamic system (4) is noninteracting with respect to the regulated outputs $\mathbf{e}_{u c}, \mathbf{e}_{t i}$ and $\mathbf{e}_{q r}$ if there exists a partition $\tau_{u c}, \tau_{t i}$ and $\tau_{q r}$ of the input vector $\tau$ such that for zero initial condition, each input $\tau_{f, 1}$ (with all the other inputs, identically zero) only affects the corresponding output $e_{(1)}$

The following theorem shows that the noninteraction of the regulated outputs $\mathbf{e}_{u c}, \mathbf{e}_{t i}$ and $\mathbf{e}_{q r}$ for the dymamic system (4), is an intrinsic structural property of general (non-indeterminate) manipulation systems.

Assume that:

H1: The manipulation mechanism is not indeterminate, $\operatorname{ker}\left(\mathbf{G}^{T}\right)=\mathbf{0}$ :

H2: $\operatorname{im}\left(\boldsymbol{\Gamma}_{q c}\right)$ is $\mathbf{M}_{h}^{T} \mathbf{J}^{T} \mathbf{K J}$-invariant and $\operatorname{im}\left(\boldsymbol{\Gamma}_{u c}\right)$ is $\mathbf{M}_{o}^{T} \mathbf{G} \mathbf{K} \mathbf{G}^{T}$-invariant,

then, the following theorem holds

Theorem 2 (Noninteraction) Consider the linearized manipulation system. (4). Under the hypothesis H1. there exists a noninteracting control law decoupling the following outputs:

a) rigid-body object motions $\mathbf{e}_{u c \text { : }}$ :

b) reachable internal forces $\mathbf{e}_{t i}$,

c) mechanism redundancy $\mathbf{e}_{q r}$;

Remark 1 The technical hypothesis $\mathbf{H} 2$ is introduced in order to simplify the proof of the theorem.

Remark 2 In $[8]$ the authors discussed the consistency of the outputs chosen in Theorem 2, where for consistency we mean that the whole set of outputs is pointwise controllable and that it directly accommodates for specification of manipulation tasks. Moreover, in [8] it was shown that these outputs, $\mathbf{e}_{\imath c}, \mathbf{e}_{t i}$ and $\mathbf{e}_{q r}$, are functionally controllable and exhaust the control capabilities, i.e. the input-output representation is invertible and square. In this paper we show that there always exists an observer-based control law which is locally noninteracting with respect to these outputs. The theorem on noninteraction is stated for the whole class of general (non-indeterminate) manipulation systems, i.e. whatever be the Jacobian and grasp matrices (with $\operatorname{ker}\left(\mathbf{G}^{T}\right)=0$ ) but, as pointed out elsewhere, we underline the relevance of Theorem 2 to the subclass of defective system characterized by a low number of control variables.

\section{Noninteraction: sketch of the proof}

In this section the proof of the main result is sketched. It is based on the analysis of the system-theoretic structural properties of manipulation systems, summarized in a standard form for the linearized dynamics, given in [5] and [9].

A detailed proof of the Noninteraction Theorem, is given in [10].

Under the hypothesis $\mathbf{H 1}$, the couple $\left(\mathbf{A}, \mathbf{B}_{\tau}\right)$ is stabilizable (cf. Proposition 1) and under $\mathbf{H} 2$ the linearized system (4) is detectable from the informative output $\mathbf{y}=\left(\mathbf{q}^{T}, \mathbf{t}^{T}\right)^{T}$ (cf. [5]). Then, according to [2], there exists an observer-based controller noninteracting with respect to the regulated outputs $(9),(10)$ and (11):

$$
\begin{aligned}
& \mathbf{e}_{u c}=\mathbf{E}_{u c} \mathbf{x}=\left[\begin{array}{llll}
0 & \Gamma_{u c}^{P} & 0 & 0
\end{array}\right] \mathbf{x} \\
& \mathbf{e}_{t i}=\mathbf{E}_{t i} \mathbf{x}=\left[\begin{array}{llll}
\mathbf{Q} & 0 & \mathbf{Q} & 0
\end{array}\right] \mathbf{x} ;
\end{aligned}
$$

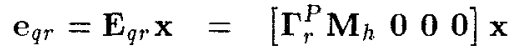

if and only if

$$
\begin{array}{ll}
\text { a) } & \mathbf{E}_{u c} \mathcal{R}_{\mathcal{K}_{u c}}=\operatorname{im}\left(\mathbf{E}_{u c}\right) ; \\
\text { b) } & \mathbf{E}_{t i} \mathcal{R}_{\mathcal{K}_{t i}}=\operatorname{im}\left(\mathbf{E}_{t i}\right) \\
\text { c) } & \mathbf{E}_{q r} \mathcal{R}_{\mathcal{K}_{q r}}=\operatorname{im}\left(\mathbf{E}_{q r}\right) ;
\end{array}
$$

where

$$
\begin{aligned}
& \mathcal{K}_{u c}=\operatorname{ker}\left(\mathbf{E}_{t i}\right) \cap \operatorname{ker}\left(\mathbf{E}_{q r}\right) \\
& \mathcal{K}_{t i}=\operatorname{ker}\left(\mathbf{E}_{u c}\right) \cap \operatorname{ker}\left(\mathbf{E}_{q r}\right) \\
& \mathcal{K}_{q r}=\operatorname{ker}\left(\mathbf{E}_{t i}\right) \cap \operatorname{ker}\left(\mathbf{E}_{u c}\right)
\end{aligned}
$$

By $\mathcal{R}_{\mathcal{K}_{(\cdot)}}$ we denote the $\mathcal{K}_{(\cdot)}$-constrained controllability subspace which is the subspace of all the points reachable through trajectories leaving the origin and belonging to $\mathcal{K}_{(\cdot)}$.

In what follows we focus on equalities (16) and to simplify the proof, we replace the intersection subspaces $\mathcal{K}_{u c}, \mathcal{K}_{t i}$ and $\mathcal{K}_{q r}$ in (17) with suitable subspaces $\operatorname{im}\left(\mathbf{B}_{u c}\right), \operatorname{im}\left(\mathbf{B}_{t i}\right)$ and $\mathrm{im}\left(\mathbf{B}_{q r}\right)$ whose constrained controllability sets do suffice for complete noninteracion.

Proof of a) It can be shown that

$$
\begin{aligned}
\mathcal{K}_{u c} \supseteq \operatorname{im}\left(\mathbf{B}_{u c}\right) & \text { with } \\
\mathbf{B}_{u c} & =\left[\begin{array}{cccc}
\boldsymbol{\Gamma}_{q c} & \mathbf{0} & \boldsymbol{\Gamma}_{q c} & \mathbf{0} \\
\boldsymbol{\Gamma}_{u c} & \mathbf{0} & -\boldsymbol{\Gamma}_{u c} & \mathbf{0} \\
\mathbf{0} & \boldsymbol{\Gamma}_{q c} & \mathbf{0} & \boldsymbol{\Gamma}_{q c} \\
\mathbf{0} & \boldsymbol{\Gamma}_{u c} & \mathbf{0} & -\boldsymbol{\Gamma}_{u c}
\end{array}\right] ;
\end{aligned}
$$

Clearly $\mathcal{R}_{\mathbf{B}_{u c}}$, the $\mathbf{B}_{u c}$-constrained controllability subspace, is only a subset of $\mathcal{R}_{\mathcal{K}_{u c}}$ but it will suffice to prove a).

In order to evaluate $\mathcal{R}_{\mathbf{B}_{u c}}$ we must face the iterative nature of the algorithms (see [2]) computing the maximal controlled invariants $\max \mathcal{V}($.$) 's and the minimal$ conditioned invariants $\min \mathcal{S}(\cdot)$ 's. In fact the minimal self-bounded controlled invariant $\mathcal{R}_{\mathbf{B}_{u c}}$ is computed as follows $\mathcal{R}_{\mathbf{B}_{u c}}=\max \mathcal{V}\left(\mathbf{A} \cdot \operatorname{im}\left(\mathbf{B}_{\tau}\right), \operatorname{im}\left(\mathbf{B}_{u r}\right)\right) \cap$ $\min \mathcal{S}\left(\mathbf{A}, \operatorname{im}\left(\mathbf{B}_{u c}\right), \operatorname{im}\left(\mathbf{B}_{\tau}\right)\right)$.

After some algebraic manipulations reported, we obtain that

$$
\mathcal{R}_{\mathbf{B}_{u c}} \supseteq \operatorname{im}\left[\begin{array}{cc}
\boldsymbol{\Gamma}_{q c} & 0 \\
\boldsymbol{\Gamma}_{u c} & 0 \\
0 & \boldsymbol{\Gamma}_{q c} \\
0 & \boldsymbol{\Gamma}_{u \odot}
\end{array}\right]
$$


To complete the proof it remains to verify that $\mathbf{E}_{u c} \mathcal{R}_{\mathbf{B}_{u c}}=\operatorname{im}\left(\mathbf{E}_{u c}\right)$ and this is trivial since from (9) $\mathbf{E}_{u c}=\Gamma_{u c}\left(\boldsymbol{\Gamma}_{u c}^{T} \boldsymbol{\Gamma}_{u c}\right)^{-1}\left[\begin{array}{llll}\mathbf{0} & \boldsymbol{\Gamma}_{u c}^{T} & \mathbf{0} & 0\end{array}\right]$

Proof of b) Analogously,

$$
\begin{aligned}
& \mathcal{K}_{t i} \supseteq \operatorname{im}\left(\mathbf{B}_{t i}\right) \quad \text { with } \\
& \mathbf{B}_{t i}=\left[\begin{array}{cccccc}
\boldsymbol{\Gamma}_{h} & \mathbf{0} & \mathbf{S}_{q} & \mathbf{0} & \mathbf{0} & \mathbf{0} \\
\mathbf{0} & \mathbf{0} & \mathbf{0} & \mathbf{0} & \mathbf{P} & \mathbf{0} \\
\mathbf{0} & \boldsymbol{\Gamma}_{h} & \mathbf{0} & \mathbf{S}_{q} & \mathbf{0} & \mathbf{0} \\
\mathbf{0} & \mathbf{0} & \mathbf{0} & \mathbf{0} & \mathbf{0} & \mathbf{P}
\end{array}\right],
\end{aligned}
$$

where $\mathbf{P}=\operatorname{ker}\left(\boldsymbol{\Gamma}_{u c}^{T}\right) \cap \mathbf{S}_{u}$ and $\mathbf{S}_{q}$ is the b.m. of $\min \mathcal{I}\left(\mathbf{M}_{h}^{-1} \mathbf{J}^{T} \mathbf{K} \mathbf{J}, \mathbf{M}_{h}^{-1} \mathbf{J}^{T} \mathbf{K} \mathbf{G}^{T}\right)$ while $\mathbf{S}_{u}$ is the b.m. of $\min \mathcal{I}\left(\mathbf{M}_{o}^{-1} \mathbf{G K} \mathbf{G}^{T}, \mathbf{M}_{o}^{-1} \mathbf{G K J}\right)$.

As before, $\mathcal{R}_{\mathbf{B}_{t i}}=\max \mathcal{V}\left(\mathbf{A}, \operatorname{im}\left(\mathbf{B}_{r}\right), \operatorname{im}\left(\mathbf{B}_{t i}\right)\right) \cap$ $\min \mathcal{S}\left(\mathbf{A}, \operatorname{im}\left(\mathbf{B}_{t i}\right), i \mathrm{im}\left(\mathbf{B}_{\tau}\right)\right)$ is a subset of $\mathcal{R}_{\mathcal{K}_{t i}}$ but it will suffice for this proof. We obtain (cf. [10]) that

$$
\mathcal{R}_{\mathbf{B}_{t i}} \supseteq \operatorname{im}\left[\begin{array}{cccc}
\boldsymbol{\Gamma}_{h} & \mathbf{0} & \mathbf{S}_{q} \mathbf{Z} & \mathbf{0} \\
\mathbf{0} & \mathbf{0} & \mathbf{0} & \mathbf{0} \\
\mathbf{0} & \boldsymbol{\Gamma}_{h} & \mathbf{0} & \mathbf{S}_{q} \mathbf{Z} \\
\mathbf{0} & \mathbf{0} & \mathbf{0} & \mathbf{0}
\end{array}\right],
$$

where $\mathbf{Z}=\operatorname{ker}\left(\boldsymbol{\Gamma}_{u c}^{T} \mathbf{M}_{o}^{-1} \mathbf{G} \mathbf{K J} \mathbf{S}_{q}\right)$. To complete the proof we must verify that $\mathbf{E}_{t i} \mathcal{R}_{\mathbf{B}_{t i}}=\operatorname{im}\left(\mathbf{E}_{t i}\right)$, which is equivalent to $\operatorname{im}\left(\mathbf{Q}\left[\begin{array}{ll}\boldsymbol{\Gamma}_{h} & \mathbf{S}_{q} \mathbf{Z}\end{array}\right]\right)=\mathrm{im}(\mathbf{Q})$, proven in $[10]$.

Proof of c) Condition c) is the easiest to prove. In fact

$$
\mathcal{K}_{q r} \supseteq \operatorname{im}\left[\begin{array}{cc}
\boldsymbol{\Gamma}_{q r} & \mathbf{0} \\
\mathbf{0} & \mathbf{0} \\
\mathbf{0} & \boldsymbol{\Gamma}_{q r} \\
\mathbf{0} & \mathbf{0}
\end{array}\right]
$$

and since from (12)

$$
\mathbf{E}_{q r}=\boldsymbol{\Gamma}_{q r}\left(\boldsymbol{\Gamma}_{q r}^{T} \boldsymbol{\Gamma}_{q r}\right)^{-1}\left[\begin{array}{lllll}
\boldsymbol{\Gamma}_{q r}^{T} \mathbf{M}_{h} & \mathbf{0} & \mathbf{0} & \mathbf{0}
\end{array}\right]
$$

condition c) is verified and the proof of c) ends.

\section{Conclusions}

In this paper we considered the problem of controlling general manipulation systems. Due to the presence of defective manipulators as a relevant subclass of the general ones, the choice of the regulated outputs requires a particular attention. In fact in defective manipulators, contact forces are not completely reachable and this involves a certain complexity in controlling the whole system.

After characterizing the system outputs as the rigidbody object motions, the reachable contact forces and the possible mechanism redundancy, we focused on the problem of force/motion noninteracting control.

The geometric approach is used throughout the paper whose main result states that there always exists an observer-based control law that is locally noninteracting with respect to the aforementioned outputs.

Notice that the local force/motion noninteraction can be considered as a structural property of manipulation systems.

\section{References}

[1] Basile G. and Marro G. "A state space approach to non-interacting controls, "Ricerche di Automatica, vol. 1, n. 1, pp. 68-77, 1970.

[2] Basile G. and Marro G. Controlled and conditioned invariants in linear system theory, New Jersey, Prentice Hall, 1992

[3] A. Bicchi, "Force distribution in multiple wholelimb manipulation," in Proc. IEEE Int. Conf. Robotics Automat., 1993.

[4] A. Bicchi, C. Melchiorri and D. Balluchi, "On the mobility and manipulability of general multiple limb robots," IEEE Trans. on Robotics and Automat., vol. 11, n. 2, 1995 .

[5] A. Bicchi and D. Prattichizzo, "A standard form for the dynamics of general manipulation systems," in Proc. IEEE Int. Conf. Robotics and Automat., 1995.

[6] L. Meirovitch, Analytical methods in vibrations, Macmillan Pub. Co., Inc. New York. 1967.

[7] A.S. Morse and W.M. Wonham, "Decoupling and pole assignment by dynamic compensation," SIAM J. Control, vol. 8, n. 3, pp. 317-337, 1970.

[8] D. Prattichizzo and A. Bicchi, "Specifying consistent control goals for kinematically defective manipulation systems," in IEEE Int. Conf. Robotics Automat., 1996. In Press.

[9] D. Prattichizzo, "Structural properties and control of robotics manipulation," $P h D$ Thesis, University of Pisa, 1995

[10] D. Prattichizzo, P. Mercorelli, A. Bicchi and A. Vicino, "Noninteracting Force/Motion Control in General Manipulation Systems," Internal Report n. 40396 , Università di Pisa, Italia, 1996.

[11] J.K. Salisbury and B. Roth, "Kinematic and force analysis of articulated mechanical hands," $J$. Mech. Transm. Automat. in Des., vol. 105, 1983

[12] R.M. Murray, Z. Li and S.S. Sastry, A mathematical introduction to robotic manipulation, Boca Raton, Florida, CRC Press, 1994.

[13] W.M. Wonham, Linear multivariable control: a geometric approach, New York, Springer-Verlag, 1979.

[14] W.M. Wonham and A.S. Morse, "Decoupling and pole assignment in linear multivariable systems: a geometric approach," SIAM J. Control, vol. 8, no. 1, pp. $1-18,1970$ 